\section{Tendencias actuales sobre las infecciones asociadas al uso de catéter venoso central}

\section{Current trends on infections associated} with the use of a central venous catheter

Ingrid Verónica Ostaiza Véliz

Médico general, Centro de Salud El Cisne II Tipo

C, ingridmd28@hotmail.com,

https://orcid.org/0000-0002-7998-1264

Camilo Alberto Mackliff Arellano

Médico general, Hospital Clínica Kennedy,

drcamilomackliff@hotmail.com,

https://orcid.org/0000-0002-4400-6100

Edwin Rommel Llanos Oquendo

Médico general, Hospital Básico José Cevallos

Ruiz,romel_90@hotmail.com,

https://orcid.org/0000-0001-8410-7312

María José Agusto Álvarez

Magister en Gerencia Hospitalaria, Hospital

General del Norte de Guayaquil Los Ceibos,

magusto2019@hotmail.com,

https://orcid.org/0000-0003-4068-3080

Guayaquil - Ecuador
http://www.jah-journal.com/index.php/jah

Journal of American health

E-1

Esta obra está bajo una Licencia Creative Commons

Atribución-NoComercial-Compartirlgual 4.0 Internacional.

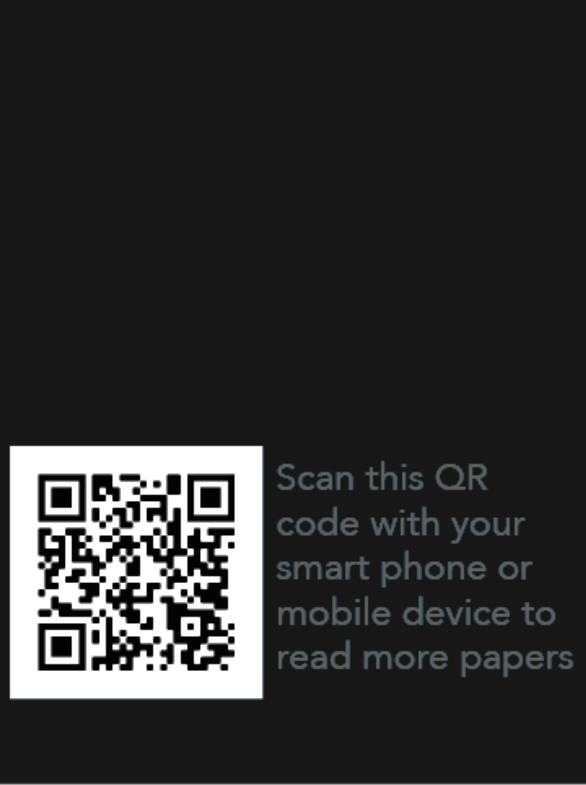

\section{RESUMEN}

Los catéteres venosos centrales (CVC), aunque indispensables en el tratamiento médico actual y de cuidados intensivos, también ponen a los pacientes en riesgo de infección relacionada con el catéter (IRC), lo que aumenta la morbilidad y la mortalidad. Se realizó un estudio para actualizar los conocimientos básicos sobre incidencia, factores de riesgo y el perfil bacteriológico de la infección del torrente sanguíneo asociada a catéter venoso central en pacientes de las unidades de cuidados intensivos. Los motores de búsqueda incluyeron Scopus, Pubmed y MEDLINE, se emplearon operadores booleanos para seleccionar las investigaciones específicas publicadas desde el 1 de enero del 2010 al 31 de diciembre del 2020.

PALABRAS CLAVE: infección del torrente sanguíneo, catéter venoso central, complicaciones, factores de riesgo.

\section{ABSTRACT}

Central venous catheters (CVCs), while indispensable in current medical and intensive care treatment, also put patients at risk for catheter-related infection (CRI), increasing morbidity and mortality. A study was carried out to update the basic knowledge on incidence, risk factors, and the bacteriological profile of bloodstream infection associated with central venous catheters in patients in intensive care units. Search engines included Scopus, Pubmed and MEDLINE, Boolean operators were used to select specific research published from January 1, 2010 to December 31, 2020. 
KEY WORDS: bloodstream infection, central venous catheter, complications, risk factors.

\section{INTRODUCCIÓN}

El acceso venoso central juega un papel importante en el manejo de pacientes críticamente enfermos y también pone a los pacientes en riesgo de diversas complicaciones iatrogénicas, incluida la infección del torrente sanguíneo asociada al catéter venoso central (ITS-CVC) $(1,2)$. La tasa de ITS-CVC en los hospitales de Estados Unidos es de 0,8/1000 días de catéter, citando la necesidad de programas efectivos de control de infecciones que incluyan políticas de vigilancia y antibióticos (3). Se estima que cada año se producen 250.000 infecciones del torrente sanguíneo y la mayoría están relacionadas con la presencia de dispositivos intravasculares. Los ITS-CVC conducen a estadías hospitalarias prolongadas y aumentan los costos de atención médica y la mortalidad.

La inserción de un catéter venoso central (CVC) es un procedimiento invasivo común $y$, a menudo, inevitable en la atención médica de pacientes críticamente enfermos (1). El CVC se utiliza para administrar medicamentos, líquidos, hemoderivados, nutrición parenteral total y para la monitorización hemodinámica. Dado que estos catéteres tienen acceso al torrente sanguíneo, pueden provocar complicaciones importantes, sobre todo una infección del torrente sanguíneo (ITS) (3). La infección del torrente sanguíneo asociada a la vía central se encuentra entre una de las infecciones asociadas a la atención médica que se pueden prevenir, lo que resulta en aumentos en la duración de las estadías hospitalarias y los costos.
Los cocos grampositivos son responsables de al menos dos tercios de las infecciones, seguidos de los bacilos gramnegativos, que son responsables de una mayor proporción de infecciones relacionadas con el catéter (IRC) en la unidad de cuidados intensivos (UCl) que en los pacientes que no están en la $\mathrm{UCl}(1,2,3)$. La formación de biopelículas en los catéteres no solo se ha implicado como un factor importante involucrado en la infección relacionada con el dispositivo, sino que también confiere resistencia al tratamiento antimicrobiano (4). Se ha demostrado que las estrategias preventivas como el uso de catéteres impregnados con antimicrobianos y, sobre todo, las técnicas de inserción de catéteres asépticos con precauciones de barrera estéril máxima reducen la tasa de ITS-CVC (5).

La condición de los pacientes con ITS en las unidades de cuidados intensivos (UCI) ha mejorado después de la implementación de un paquete de inserción y mantenimiento de una vía central. La incidencia de la infección es mayor en los países en desarrollo que en los países desarrollados. Las altas tasas de ITS-CVC en las $\mathrm{UCl}$ se han atribuido al escaso cumplimiento de las prácticas de control de infecciones. En la UCl, la vía central puede ser necesaria durante períodos prolongados, especialmente en pacientes con enfermedades crónicas, ya que se manipula varias veces y conduce a la colonización con la flora hospitalaria. Además, algunos catéteres pueden insertarse en situaciones urgentes, durante las cuales pueden verse comprometidas las precauciones asépticas óptimas.

El objetivo de la presente investigación es proporcionar a la comunidad científica 
una actualización de los referentes teóricos más importantes de las infecciones asociadas al uso del catéter venoso central, que ayude en la toma de decisiones y en la implementación de medidas de prevención basada en los factores de riesgo.

\section{MATERIALES Y MÉTODOS}

Se realizó una búsqueda en la base de datos de MEDLINE, PubMed y Scopus. Se incluyeron los estudios que informaron la tasa de ITS-CVC en pacientes con hospitalizados en unidades de cuidados intensivos, estudios tipo ensayos, de análisis de datos y metaanálisis, además de publicaciones desde el 1 de enero del 2010 al 31 de diciembre del 2020. Se emplearon palabras claves para la identificación de los artículos como: infección del torrente sanguíneo, catéter venoso central, complicaciones, factores de riesgo, las cuales fueron combinadas con el uso de operadores boleanos de intersección (AND), unión (OR) y exclusión (NOT).

\section{RESULTADOS}

La inserción de un catéter venoso central (CVC) es un procedimiento invasivo común $y$, a menudo, inevitable en la atención médica de pacientes críticamente enfermos. Dado que estos catéteres tienen acceso al torrente sanguíneo, pueden provocar complicaciones importantes, sobre todo una infección del torrente sanguíneo (ITS) (1). La infección del torrente sanguíneo asociada a la vía central (ITS-CVC) se encuentra entre una de las infecciones asociadas a la atención médica que se pueden prevenir, lo que resulta en aumentos en la duración de las estadías hospitalarias y los costos $(5,6,7)$.

\section{Definición de acceso venoso central}

Para estas pautas, el acceso venoso central se define como la colocación de un catéter de manera que el catéter se inserte en un gran vaso venoso. Los grandes vasos venosos incluyen vena cava superior, vena cava inferior, venas braquiocefálicas, venas yugulares internas, venas subclavias, venas ilíacas y venas femorales comunes. Se excluyen los catéteres que terminan en una arteria sistémica (1). El acceso venoso central es necesario para una variedad de situaciones para el cateterismo temporal y permanente. El acceso subclavio puede ser una técnica segura y confiable para lograr acceso venoso central. El acceso subclavio es utilizado a diario por muchos profesionales y es una habilidad valiosa para cualquier proveedor de atención médica (9).

\section{Indicaciones}

El cateterismo venoso central proporciona una ruta para la administración de soluciones cáusticas o críticas, medicamentos y permite la medición de la presión venosa central. Las indicaciones para el acceso venoso central incluyen (1):

- Inadecuado acceso venoso periférico.

- Administración de medicamentos nocivos para las venas periféricas

- Monitorización hemodinámica avanzada

- Acceso cardíaco para la estimulación transvenosa temporal.

- Hemodiálisis.

- Oxigenación por membrana extracorpórea.

\section{Contraindicaciones}

Debido a que el CVC puede salvar vidas, no hay contraindicaciones absolutas. Las 
contraindicaciones generales para la colocación de un catéter venoso central incluyen infección del área que cubre la vena objetivo y trombosis de la vena. Las contraindicaciones específicas del sitio $y$ relativas incluyen coagulopatía, aunque esto no es una contraindicación absoluta. Se debe tener extremo cuidado en pacientes con coagulopatía y en otros pacientes para quienes las complicaciones serían potencialmente mortales (2). Aunque la coagulopatía aumenta el riesgo de hemorragia durante el CVC, con una cuidadosa selección del sitio y una técnica meticulosa, las complicaciones hemorrágicas pueden mantenerse al mínimo. Debido a que la vena subclavia y la arteria no son accesibles para la compresión directa, el sitio subclavio es el menos apropiado para el paciente con una diátesis hemorrágica (2).

Las limitaciones anatómicas, como la obesidad mórbida, hacen que el CVC sea más difícil y peligroso. Cuando sea posible, los catéteres no deben colocarse a través del sitio de quemadura cutánea o infección. El riesgo de neumotórax durante el CVC aumenta con la hiperinflación de los pulmones asociada con la enfermedad pulmonar obstructiva crónica o la ventilación mecánica con grandes volúmenes corrientes o con un aumento de la presión positiva al final de la espiración (2). La trombosis venosa en un sitio de cateterismo planificado puede dictar la selección de sitios alternativos. EI traumatismo abdominal penetrante o la disrupción de la vena cava inferior conocida hacen que la canulación venosa femoral sea menos atractiva. Un paciente informado y tranquilo facilita un CVC seguro. Cuando sea posible, se debe obtener el consentimiento informado antes del cateterismo (3).

\section{Selección del catéter}

Una gran variedad de catéteres venosos centrales está disponible para uso clínico. Pueden tener lúmenes únicos o múltiples. El número de lúmenes del catéter no afecta la tasa de complicaciones, por lo que se debe elegir el número de lúmenes para satisfacer mejor las necesidades clínicas. Los catéteres multilumen son comúnmente seleccionados y a menudo niegan la necesidad de múltiples sitios CVC (4). Los catéteres de triple luz y cuádruple lumen son extremadamente útiles en la atención diaria de pacientes críticamente enfermos. Sin embargo, debido al diámetro individual relativamente pequeño y la longitud del catéter larga $(20-30 \mathrm{~cm})$, la resistencia al flujo es alta, lo que hace que estos catéteres sean menos que ideales para la infusión rápida de líquidos (5).

\section{Tipos de catéteres}

\section{Catéteres no tunelizados}

Los catéteres no tunelizados se usan comúnmente en situaciones de emergencia ya que es fácil de colocar y no necesita habilidades quirúrgicas especiales para la inserción. Como la duración del uso de este catéter es corta, el catéter debe ser removido tan pronto como sea posible $(8,10)$.

\section{Catéteres tunelizados}

Los catéteres tunelizados se usan cuando se necesita el acceso a la corriente sanguínea de un paciente por múltiples veces durante un período de más de 30 días. Los catéteres tunelizados pueden ser esposados 0 sin manguitos. En comparación con los catéteres no 
tunelizados, causan menos complicaciones infecciosas $(8,10)$.

\section{Puertos implantados}

Los puertos implantados son adecuados para uso a largo plazo, causan menos infecciones, complicaciones y rinden mejores resultados cosméticos en comparación con los catéteres antes mencionados. Sin embargo, requieren cirugía de implantación con un alto costo $(7,8)$.

Catéteres de diálisis y catéteres centrales insertados periféricamente (PICC)

Los catéteres de diálisis tienen orificios grandes que requieren heparinización para prevenir la coagulación mientras que los PICC generalmente se insertan en una vena periférica en el área antecubital (1).

\section{Sitios de colocación del catéter}

Los tres sitios más comunes para el acceso venoso central son vena subclavia, vena yugular interna y vena femoral. El enfoque más común para el cateterismo de la vena subclavia es el abordaje infraclavicular. El operador se encuentra al mismo lado de la vena y gira la cabeza del paciente en la dirección opuesta. La piel se perfora alrededor de $1 \mathrm{~cm}$ caudal a la unión de los tercios medio y medio de la clavícula. Apunta la aguja hacia la muesca esternal $(1,7,8)$.

El operador se localiza a la cabeza de la cama y gira la cabeza del paciente hacia el lado opuesto del sitio de destino. El triángulo entre las porciones medial y lateral del músculo esternocleidomastoideo se identifica y la punción se hace en el ápice del triángulo. La aguja apunta hacia el pezón ipsilateral $(7,8)$.
La vena yugular derecha va directamente a la vena cava superior, mientras que el vértice del pulmón izquierdo es ligeramente más alto que el derecho, lo que aumenta el riesgo de neumotórax. Por lo tanto, la vena yugular derecha es más comúnmente accedida que la izquierda $(7,9)$. El punto de referencia está a medio camino entre la espina ilíaca anterosuperior y el tubérculo púbico donde se localiza la arteria femoral. La vena femoral es ligeramente medial a la arteria. $\mathrm{EI}$ sitio ideal debe tener menos complicaciones infecciosas, trombóticas y mecánicas con una mayor tasa de éxito de inserción. Comparación entre vena subclavia, vena yugular y vena femoral $(4,9)$.

\section{Infecciones relacionadas con el catéter}

\section{Definición}

Bacteriemia/fungemia en un paciente con un catéter intravascular con al menos un hemocultivo positivo obtenido de una vena periférica, manifestaciones clínicas de infecciones (es decir, fiebre, escalofríos y / o hipotensión) y sin una fuente aparente de ITS excepto el catéter. Debe estar presente uno de los siguientes: un cultivo positivo semicuantitativo (> 15 UFC/segmento de catéter) o cuantitativo (> 102 UFC/segmento de catéter) mediante el cual el mismo organismo (especie $y$ antibiograma) se aísla del segmento del catéter y de la sangre periférica (11).

Las infecciones por catéter se producen por medio de uno de estos tres mecanismos: inserción local en el sitio de infección, que viaja por el catéter externamente; o colonización del eje seguido por infección a través de la vía intraluminal o siembra por vía hematógena 
del catéter $(2,6)$. El Instituto para la Mejora de la Salud recomienda cinco pasos para reducir infecciones de la línea central: higiene de las manos, adherencia a las precauciones de barrera máximas, antisepsia de la piel con clorhexidina, selección del sitio óptimo del catéter y revisión diaria del catéter, con pronta eliminación cuando el catéter ya no es necesario (12).

Se ha demostrado concluyentemente que la implementación de estos pasos disminuye la tasa de infección del torrente sanguíneo relacionada con el catéter. El cambio programado de un catéter sobre una guía o mover un catéter a un sitio nuevo puede aumentar la resistencia mecánica y complicaciones infecciosas y ninguno es recomendado. Se ha demostrado que los catéteres impregnados de antimicrobianos disminuyen la tasa de infecciones del torrente sanguíneo relacionadas con el catéter. Antibiótico tópico; los ungüentos son ineficaces, promueven bacterias resistentes a los antibióticos y aumentan los hongos colonización $(2,12,13)$.

Los catéteres impregnados con clorhexidina y sulfadiazina de plata y los catéteres impregnados con minociclina están asociados con menos infecciones del torrente sanguíneo relacionadas con el catéter que los catéteres no impregnados. Los catéteres impregnados deben considerarse en todos los casos, especialmente cuando la tasa de infección relacionada con el catéter institucional supera el $2 \%(2,6,13)$.

El uso del sitio subclavio se asocia con menos infecciones relacionadas con el catéter que los sitios yugular interno o femoral, lo que lo convierte en un sitio preferido en muchos pacientes. Se ha demostrado que la aplicación de una pomada antibiótica en el sitio de la punción de la piel aumenta la tasa de colonización fúngica y promueve el crecimiento de bacterias resistentes a los antibióticos. Por lo tanto, los ungüentos antibióticos no deben usarse. El uso de manguitos impregnados con plata subcutánea es controvertido, pero no se ha asociado con una reducción en las infecciones del torrente sanguíneo relacionadas con el catéter $(1,2,13)$.

Por lo tanto, no se pueden hacer recomendaciones firmes sobre los apósitos y los cambios de apósito en este momento. Las investigaciones no respaldan la extracción y el reemplazo sistemático del catéter de forma programada. Los catéteres venosos centrales deben retirarse tan pronto como ya no los necesiten (14). Los intercambios rutinarios de catéteres sobre una guía no son recomendables. Se debe extraer el catéter y se debe insertar un nuevo catéter si el médico sospecha la posibilidad de infección relacionada con el catéter debido a toxicidad sistémica o purulencia en el sitio de inserción del catéter.

Existen varios sistemas de definición y clasificación de las infecciones del catéter, lo que genera gran controversia. Los 2 sistemas principales son $(9,15)$ :

- Los criterios que se emplean para estudios epidemiológicos: bacteriemia asociada a la vía central (CLABSI) o bacteriemia asociada al catéter (CABSI).

- Los criterios para la definición clínica de infección relacionada con el catéter (IRC), incluida la bacteriemia relacionada con el catéter $(B R C)$, que 
son los utilizados en la práctica médica para diagnóstico y tratamiento de las mismas.

La infección relacionada con el catéter venoso central puede ser:

\section{A) Locales}

- Colonización del catéter: crecimiento bacteriano $>15$ UFC en un segmento de $5 \mathrm{~cm}$ de punta de catéter por cultivo semicuantitativo, o crecimiento $>100$ UFC de un catéter por técnica cuantitativa, sin que existan signos clínicos de infección

- Infección del punto de entrada: signos clínicos de infección local: eritema, calor, induración, pus; documentada microbiológicamente: si cultivo positivo del punto de entrada del catéter, con hemocultivo negativo (16).

\section{B) Sistémicas}

- Bacteriemia relacionada con el catéter (BRC) (ver apartado "diagnóstico/cultivos")

- Bacteriemia relacionada con los líquidos de infusión: clínica de sepsis, con aislamiento del mismo microorganismo en los líquidos de infusión y en el hemocultivo periférico (16).

\section{c) Complicadas}

- Bacteriemia persistente: crecimiento del mismo germen en hemocultivo > $72 \mathrm{~h}$ tras inicio de antibioterapia adecuada

- Trombosis séptica

- Endocarditis

- Otras metástasis a distancia: ej. Osteomielitis (16)

\section{Mecanismo}

de

infección

microbiología

La colonización del catéter puede ocurrir por dos vías principales: la vía extraluminal - la vía intraluminal. La colonización del catéter desde su superficie dérmica es la ruta predominante de colonización para los CVC a corto plazo ( $<15-30$ días), mientras que la colonización por vía intraluminal resultante de una contaminación del concentrador predomina para los CVC a largo plazo. Se debe tener en cuenta a la hora de elegir entre diferentes opciones de diagnóstico y estrategias preventivas $(1,20,22,23)$.

La aparición de bacteriemia causada por organismos cutáneos comunes (estafilococos coagulasa negativos [CoNS] o Staphylococcus aureus) es un criterio importante para el diagnóstico de ITS-CVC, aunque los microorganismos gramnegativos se observan cada vez más en los últimos años, especialmente en caso de sitio de inserción femoral $(15,19,20,25)$.

\section{Factores de riesgo}

- Edad: $<2$ meses

- Peso $<8 \mathrm{Kg}$

- Enfermedad de base y su evolución clínica

- Neutropenia

- Nutrición parenteral completa

- Ventilación mecánica

- Ingreso en UCIP

- Infecciones preexistentes

- Dispositivos implantados

- Tiempo de permanencia del catéter (> 7 ○ 12 días en percutáneos, según diferentes estudios)

- Catéteres centrales de inserción percutánea (frente a los epicutáneos 
centrales, tunelizados o los reservorios de implantación completa)

- Catéteres de mayor número de luces (no demostrado en pediatría, en que incluso podría aconsejarse mayor número para evitar manipulación de las luces con cambios constantes de medicación)

- Catéteres impregnados de antibiótico o solución antiséptica, menor riesgo

- Lugar de inserción: en pediatría no existe relación, frente al menor riesgo de la localización en subclavia en pacientes adultos. En pacientes pediátricos, los catéteres femorales presentan una baja incidencia de complicaciones mecánicas y su tasa de infección puede ser equivalente 0 incluso inferior a la de los catéteres no femorales $(17,25,26)$.

\section{Patogenia}

La superficie del catéter favorece la adhesión de las bacterias, las cuales producen a su vez una biocapa que las recubre y protege de los mecanismos de defensa del huésped y los antimicrobianos. Las vías de colonización de un catéter central pueden ser $(16,17)$ :

- Extraluminal: la flora cutánea migra desde la piel hasta la superficie intravascular del catéter, a través del manguito de fibrina que se forma tras su colocación

- Intraluminal: las bacterias alcanzan el interior del catéter desde la conexión

- Hematógena (3-10\% de los casos): los gérmenes colonizan el catéter vía sanguínea desde otro foco a distancia

- Líquidos de infusión (<3\% de los casos)

La colonización de un catéter de corta permanencia (insertado hace $<8$ días) suele realizarse vía extraluminal $(70-90 \%$ de los casos), mientras que la vía intraluminal sólo está implicada en el $10-50 \%$ de los casos; por el contrario, la intraluminal es la vía más frecuente en la infección de los catéteres de larga permanencia ( $66 \%$ versus al $25 \%$ de la extraluminal) $(17,18,26)$.

\section{Diagnóstico}

Su dificultad viene determinada porque es poco frecuente la aparición de signos inflamatorios externos y, concretamente en niños, por la dificultad de obtención de hemocultivos periféricos y de la retirada del catéter para el cultivo de la punta en los intentos de preservación del mismo (18).

\section{Clínica}

Un hemocultivo positivo extraído de CVC puede deberse únicamente a la colonización del catéter, y se requiere una interpretación clínica para determinar si existe infección, aunque la negatividad del hemocultivo sí puede ayudar a excluir bacteriemia relacionada al catéter. La clínica posee baja especificidad y sensibilidad para el diagnóstico. Existe buena correlación si la clínica desaparece en 24-48h tras la retirada del catéter. Aumenta la probabilidad de ITS-CVC si suma $(18,19)$ :

a) Clínica grave: sepsis grave o shock séptico

b) No existe evidencia de otro foco

c) Signos con probabilidad elevada de estar relacionados con la infección del catéter

- Hipotensión tras infundir por el catéter

- Signos de infección en el punto de inserción: pus, eritema o induración (o del trayecto subcutáneo en tunelizados)

Los pacientes con bacteriemia deben mejorar en las primeras 48-72h de tratamiento adecuado. La persistencia de fiebre o la reaparición de la misma o de otros datos de respuesta inflamatoria, tras $72 \mathrm{~h}$ del inicio del tratamiento, deben alertar sobre un posible curso complicado. 
También la reaparición de fiebre o signos de sepsis tras la finalización del tratamiento de una bacteriemia implica la reevaluación del paciente y el descartar una recidiva o una complicación supurada $(18,19)$.

1. Cultivos

El método más certero para el diagnóstico de ITS es el cultivo de la punta de catéter. Según un meta-análisis Ilevado a cabo al respecto, los métodos más adecuados para el diagnóstico en catéter de corta permanencia son el cultivo cuantitativo o semicuantitativo del catéter combinado con 2 hemocultivos, y para catéter de larga permanencia, 2 hemocultivos simultáneos. Teniendo en cuenta la obvia limitación que supone el depender del cultivo de la punta de catéter para el diagnóstico certero, se han desarrollado otras "técnicas conservadoras", como la diferencia proporcional del número de UFC de los cultivos cuantitativos (ratio quantitative cultures, RQC) y el tiempo diferencial de positivización $(18,19)$.

\section{DISCUSIÓN}

Los catéteres venosos centrales (CVC) se usan comúnmente en pacientes críticamente enfermos para la administración de líquidos, medicamentos, productos sanguíneos y nutrición parenteral, para la inserción de un electrodo de marcapasos transvenoso y para monitorear el estado hemodinámico $(20,21,22)$. El uso de catéteres es habitual en pacientes críticos; en el estudio EPIC, al $78 \%$ de los pacientes críticamente enfermos se les insertó algún tipo de CVC. El cateterismo venoso central puede causar diferentes complicaciones, que incluyen infección, hemorragia y trombosis (23). El interés en las infecciones relacionadas con el catéter radica en la mortalidad y los costes que representa.

El lugar de cateterismo venoso asociado con el mayor riesgo de infección sigue siendo controvertido. Varios estudios han analizado la incidencia de colonización de la punta del catéter (CTC) según diferentes accesos CVC $(18,21,22)$; en algunos estudios, se produjo una mayor incidencia con el acceso femoral; en algunos fue mayor con acceso yugular; y otros compararon solo el acceso yugular versus subclavio, encontrando una mayor incidencia en el primero. Dos estudios que analizaron la incidencia de ITS-CVC con respecto a diferentes accesos CVC encontraron una mayor incidencia con el acceso femoral $(23,24)$.

Sulek et al realizaron un ensayo controlado aleatorio que comparó el acceso venoso central yugular izquierdo y derecho mediante técnica de puntos de referencia anatómicos y acceso guiado por ultrasonografía y encontraron que el acceso venoso yugular izquierdo mostró una mayor tasa de punción arterial, hematoma y guía fallada (3) (27). Sin embargo, Parienti et al publicó un metanálisis para abordar únicamente las complicaciones infecciosas y argumentó que el sitio de la vena subclavia se asoció con menos infecciones relacionadas con el catéter en comparación con otros sitios alternativos (1.3 vs. 2.7 por 1000 días-catéter, $p \quad<0.001)$. Las infecciones relacionadas con el catéter también fueron menores en la vena subclavia cuando en comparación con la vena yugular interna (índice de densidad de incidencia $0.46,95 \% \mathrm{Cl}: 0.3 \mathrm{e} 0.7, \mathrm{I} 2 \mathrm{1} / 40 \%$ ) y vena femoral (índice de densidad de incidencia 0.27, IC 95\%: 0.15e0.48, I 2 1 1/4 31\%) (6). 
Otro análisis de dos ensayos aleatorizados publicados por Timsit et al observaron resultados similares. Aunque el estudio no mostró diferencias en la infección del torrente sanguíneo relacionada con el catéter, la infección y la colonización relacionadas con el catéter mayor fueron mayores en las mujeres ( $H R$ 0,$39 ; p 1 / 40,001$ ) y en los catéteres que estuvieron en su lugar por más de 4 días (HR $0,75 ; p 1 / 40.05)(2)(27)$.

Dezfulian et al, comparó los catéteres venosos centrales multilumen y de una sola luz y llegaron a la conclusión de que la tasa global de infección de la corriente sanguínea relacionada con el catéter (CRBSI) era mayor en los catéteres multilumen (OR 2.15, IC 95\%: 1.00e4.66), pero no hubo diferencias cuando solo se incluyeron estudios de alta calidad (7) (27).

Un metanálisis de 7 ensayos de control aleatorizados encontró que los catéteres venosos centrales impregnados con rifampicina condujeron a una reducción significativa en la tasa de CRBSI (OR 0,23, IC del 95\%: 0.14e0.40) (8). Sin embargo, otro estudio de catéteres centrales insertados periféricamente que compararon catéteres impregnados con clorhexidina (CHG) y no CHG no encontró diferencias en CRBSI entre los grupos (9). La última revisión publicada en 2016 de Cochrane indicó que el catéter venoso central impregnado con antimicrobiano redujo significativamente la CRBSI (RR 0,62; IC del 95\%: 0,52-0,74) pero no se observaron efectos para minimizar la sepsis o la mortalidad (10) (27).

Las infecciones relacionadas con CVC se asocian con $20-50 \%$ de las muertes relacionadas con nutrición parenteral (NP). La ITS-CVC es una de las complicaciones más comunes observadas en pacientes con NP porque la manipulación del catéter para la infusión o medicación proporciona una ventana de oportunidad para la contaminación del catéter, que puede progresar a ITS. Además, se ha propuesto que el contenido de las fórmulas como dextrosa, aminoácidos y emulsiones lipídicas favorecen el crecimiento microbiano si la línea está contaminada.

\section{CONCLUSIONES}

La incidencia de infección del torrente sanguíneo asociada a catéter venoso central (ITS-CVC) es variable en todos los estudios, pero sobrepasa el $15 \%$ en la mayoría. La estancia prolongada en la $\mathrm{UCl}$ y el cateterismo más prolongado son considerados los principales factores de riesgo. El S. aureus se aísla con mayor frecuencia en los casos di ITS.CVC. La amenaza de la resistencia a múltiples fármacos y la formación de biopelículas en los CVC se asocia con las ITS.CVC.

El conocimiento del perfil microbiológico y la estrategia preventiva de las ITS-CVC es fundamental para la prevención y el inicio oportuno de la terapia antiinfecciosa más adecuada, en caso de que ocurra. La vigilancia de las infecciones asociadas a la atención sanitaria es fundamental para los esfuerzos de prevención y control de infecciones. Los datos de vigilancia de infecciones se utilizan para medir el éxito de los programas de prevención y control de infecciones y para identificar áreas de mejora. Se debe llevar a cabo una vigilancia de rutina para la detección de infecciones y la implementación de las medidas 
preventivas adecuadas para brindar una atención de calidad a los pacientes. El conocimiento del perfil de susceptibilidad microbiológica y antimicrobiana es esencial para el inicio oportuno de la terapia antiinfecciosa más adecuada.

\section{REFERENCIAS}

1. Urman R EJ. Anestesia de bolsillo. Cap. 11: Cateterización venosa central. 2nd ed.: Lippincott-Wolters Kluwer; 2014.

2. Timsit et al. Jugular versus femoral short-term catheterization and risk of infection in intensive care unit patients. Causal analysis of two randomized trials. Am J Respir Crit Care Med. 2013; 188(10): p. 1232e1239.

3. Sulek C BMLE. A randomized study of left versus right internal jugular vein cannulation in adults. J Clin Anesth. 2013; 12(1): p. 142e145.

4. American Society of Anesthesiologists. Practice Guidelines for Central Venous Access. A Report by the American Society of Anesthesiologists Task Force on Central Venous Access. Anesthesiology. 2013; 116(1): p. 53973.

5. Hind et al. Ultrasonic locating devices for central venous cannulation: metaanalysis. BMJ. 2013; 21(2): p. 327-361.

6. Parienti et al. Meta-analysis of subclavian insertion and nontunneled central venous catheter-associated infection risk reduction in critically ill adults. Crit Care Med. 2013; 40(1): p. 1627 e1634.

7. Dezfulian et al. Rates of infection for single-lumen versus multilumen central venous catheters: a metaanalysis.. Crit Care Med. 2012; 31(1): p. 2385e2390.

8. Falagas et al. Rifampicin-impregnated central venous catheters: a metaanalysis of randomized controlled trials. J Antimicrob Chemother. 2014; 59: p. 359e369.

9. Storey et al. A comparative evaluation of antimicrobial coated versus nonantimicrobial coated peripherally inserted central catheters on associated outcomes: a randomized controlled trial. Am J Infect Control. 2016; 44: p. 636e641.

10. Lai et al. Catheter impregnation, coating or bonding for reducing central venous catheter-related infections in adults. Cochrane Database Syst Rev. 2016; 3: p. CD007878.

11. Kaur M. Incidence, risk factors, microbiology of venous catheter associated bloodstream infections--a prospective study from a tertiary care hospital. Indian J Med Microbiol. Vol.33(2):248-54. doi: 10.4103/02550857.153572. 2015.

12. Ataman et al. Assessment of diagnostic methods for the catheter-related bloodstream infections in intensive care units. Mikrobiyol Bul. Vol.45(1):75-85. Turkish. PMID: 21341162.. 2011.

13. Khodare A. Incidence, Microbiological Profile, and Impact of Preventive Measures on Central Line-associated Bloodstream Infection in Liver Care Intensive Care Unit. Indian J Crit Care Med. Vol.24(1):17-22. oi: 10.5005/jpjournals-10071-23325. 2020.

14. Khanna V. Evaluation of central venous catheter associated blood stream infections: a microbiological 
observational study. J Pathog. Vol.2013:936864.

doi:10.1155/2013/936864. 2013.

15. Patil et al. Central venous catheterrelated bloodstream infections in the intensive care unit. Indian J Crit Care Med. Vol.15(4):213-223. doi:10.4103/0972-5229.92074. 2011.

16. Hadzic A. Tratato de Anestesia Regional y Manejo del dolor agudo: Mc Graw Hill; 2016.

17. Merrer et al. Complications of femoral and subclavian venous catheterization in critically ill patients: a randomized controlled trial. JAMA. 2013; 286(3): p. $700 \mathrm{e} 707$.

18. Miller R. Miller Anestesia. Cap 3: Cateterización venosa central. Séptima edición ed. España: Elsevier-Saunders; 2015.

19. Lorente L. Antimicrobial-impregnated catheters for the prevention of catheter- related bloodstream infections. World J Crit Care Med. 2016; 5: p. 137e142.

20. Doerfler M KBGA. Central venous catheter placement in patients with disorders of hemostasis. Chest. 2016; 110: p. $185 \mathrm{e} 188$.

21. Chu L. Manual de Anestesiología Clínica. Cap 10: Cateterización venosa central: Elsevier; 2013.

22. Ruiz et al. Blood stream infections associated with central and peripheral venous catheters. BMC Infect Dis. Vol.19(1):841. doi: 10.1186/s12879019-4505-2. 2019.

23. Santarpia L. Central venous catheter related bloodstream infections in adult patients on home parenteral nutrition: Prevalence, predictive factors, therapeutic outcome. Clin Nutr.
Vol.35(6):1394-1398. doi: 10.1016/j.clnu.2016.03.009.. 2016.

24. Chong et al. Comparative Efficacy of Antimicrobial Central Venous Catheters in Reducing CatheterRelated Bloodstream Infections in Adults: Abridged Cochrane Systematic Review and Network Meta-Analysis. Clin Infect Dis. 2017 May 15;64(suppl_2):S131-S140.. 2017.

25. Taylor J. Prevention of central venous catheter-related infection in the neonatal unit: a literature review. J Matern Fetal Neonatal Med. Vol.28(10):1224-30. doi: 10.3109/14767058.2014.949663.. 2015.

26. Tapia R. Infección relacionada al cateter venoso central. Hospital U Madrid Montepríncipe. Dospinible en: http://secip.com/wpcontent/uploads/2018/06/InfeccionRelacionada-con-el-Cateter-VenosoCentral-v4-2012.pdf. 2018.

27. Risco A. Complicaciones de la colocación de cateter venoso central en el hospital general Martín Icaza de babahoyo en el periodo de mayo del 2015 a diciembre del 2017. Universidad de Guaayquil. Facultad de Ciencias Médicas. Tesis de grado. 2018. 
